\title{
ALOS-2 and Sentinel-1 Backscattering Coefficients for Water and Flood Detection in Nakhon Phanom Province, Northeastern Thailand
}

\author{
Tamkuan, N. and Nagai, $M$. \\ Graduate School of Sciences and Technology for Innovation, Yamaguchi University, 2-16-1, Tokiwadai, Ube, \\ Yamaguchi 755-8611, Japan \\ E-mail: nopphawan.yui@gmail.com,ntamkuan@yamaguchi-u.ac.jp,nagaim@yamaguchi-u.ac.jp \\ DOI: https://doi.org/10.52939/ijg.v17i3.1895
}

\begin{abstract}
Synthetic Aperture Radar (SAR) plays an important role in disaster management, such as in flood area monitoring, as it can withstand severe weather or the rainy season. The main objective of this study was to investigate the potential of ALOS-2 (L-band) and Sentinel-1 (C-Band) SAR data for water and flood mapping. This study used ALOS-2 and Sentinel-1 to detect water areas from pre-and mid-flood events in Nakhon Phanom Province, Thailand. Water areas were determined via the thresholding method. The study assessed different polarizations of ALOS-2 (HH, HV) and Sentinel-1 (VH, VV) characteristics, as well as non-filtered and filtered images in the detection of water areas. Water extension detection from the optical sensor (Sentinel-2) was compared with SAR results. Water detection by ALOS-2 and Sentinel-1 images were independently observed with less cloud effect and showed good agreement with Sentinel-2. ALOS-2 HH demonstrated higher accuracy (92.46\%) than ALOS-2 HV. Sentinel-1 VV provided the highest accuracy $(95.45 \%)$ among all the results analyzed. The differences between L-band and C-band results accounted for $8.8 \%$ of the study area, with major differences appearing in the paddy fields. Water and flood detection by satellite images provided not only spatial information but also temporal dimensions. The results of this study can help inform decision-making in future flood mapping and disaster management.
\end{abstract}

\section{Introduction}

Flooding is a frequent natural disaster in much of Asia (Kanta Kafle, 2017). In early August 2018, the Asian Disaster Reduction Center (ADRC) reported that heavy rain in northeastern Thailand had caused the Mekong River to overflow, inundating many provinces. Nakhon Phanom Province, the area on which the present study focuses, was severely hit, with floods affecting all 12 of its districts and more than 12,000 households (ADRC, 2019).

A quick and well-informed response is crucial to minimizing the effects of such occurrences. Hence, geospatial information has become necessary for effective disaster management (Miyazaki et al., 2015). With this in mind, emergency response, as well as post-disaster rehabilitation services, have increasingly implemented geospatial technologies (Stevens, 2008). Among the tools widely adopted in disaster observation and management efforts - such as flood monitoring - is remote sensing, which is inherently useful for monitoring the earth's surface. Sentinel Asia, a regional cooperation project among space agencies and disaster management agencies, has applied remote sensing technologies to disaster management efforts in Asia (Kaku and Held, 2013), including in the area assessed in the present study.
Optical imagery such as Landsat-8 OLI has proven successful in water body identification (Acharya et al., 2016 and Xie et al., 2016). However, clouds have been known to strongly limit the advantages of optical imagery for spatial and temporal analysis (Eberhardt et al., 2016). SAR is one remote sensing technology that can rapidly scope out a disaster-stricken area, since it has few to no limitations when faced with clouds or adverse weather conditions associated with the rainy season. Moreover, SAR sensors provide a greater frequency in both day and nighttime observation. In Asia, it has been applied to observation and emergency response efforts for disasters such as the 2016 Kumamoto earthquake in Japan (Tamkuan and Nagai, 2017 and 2019a) and the 2018 earthquake in Sulawesi, Indonesia (Tamkuan and Nagai, 2019b)

Several remote sensing methods can be utilized to detect flood areas, including thresholding and unsupervised and supervised classifications. The thresholding method has been commonly used for inundated area extraction (Nakmuenwai et al., 2017 and Duy, 2015). It is important to note that different SAR sensors have shown different backscattering behaviors (Plank et al., 2017). The threshold value 
of SAR backscattering provides information on physical properties distinguishing water from nonwater, which can be applied in flood-related assessments. Considering such aspects, the present study applied simple thresholding (the Otsu method) to evaluate the potential and behavior of each sensor as well as polarization. Selected for this study were ALOS-2 with L-band and Sentinel-1 with C-band.

More specifically, the researchers aimed to evaluate differences in polarization pertaining to ALOS-2 (HH, HV) and Sentinel-1 (VH, VV) backscattering coefficients, for flood thresholding with filter and non-filter applications, as well as the possible capabilities and differences of ALOS-2 and Sentinel-1 in terms of water and flood detection. The findings obtained on the potential of these SAR sensors could greatly benefit future decision-making in rapid flood mapping.

\section{Methodology}

\subsection{Study Area}

Flooding occurred in northeastern Thailand due to heavy rainfall and the rising waters of the Mekong River from July to August 2018. It affected many provinces in this region, especially Nakhon Phanom Province (which is adjacent to the Mukdahan, Sakon Nakhon and Bueng Kan provinces in Thailand and bordered by Laos to the northeast). The study focused on Sentinel-1 and ALOS-2 observations in the heavily affected district of Si Songkhram and some parts of the $\mathrm{Na} \mathrm{Wa}, \mathrm{Na}$ Thom, Ban Paeng, and Tha Uthen districts in Nakhon Phanom. The area comprises the Mekong River valley, and therefore mostly plains, with the main river in the northern part being the Songkhram.

According to Thailand's Land Development Department (LDD, 2015), major land use in the province had been split among paddy fields (42.1\%); perennial crops, orchards and horticulture (24.4\%); forests (14.0\%); rangeland marshes and swamps (8.4\%); water bodies (7.5\%); urban and built-up land (3.1\%); and other classifications $(0.5 \%)$, as Figure 1 shows.

\subsection{Data and Software}

ALOS-2 and Sentinel-1 images were observed in this study area as demonstrated in Table 1. The ALOS-2 images came from the dual-polarization mode (SM3). This mode consists of $\mathrm{HH}$ and HV polarizations of SAR data characteristics. The products were in Level 2.1 format by ascending orbit direction, right-side observation. Sentinel-1 images came from the Interferometric Wide Swath Mode (IW mode) by ascending observation. The IW mode consists of $\mathrm{VH}$ and $\mathrm{VV}$ polarizations.
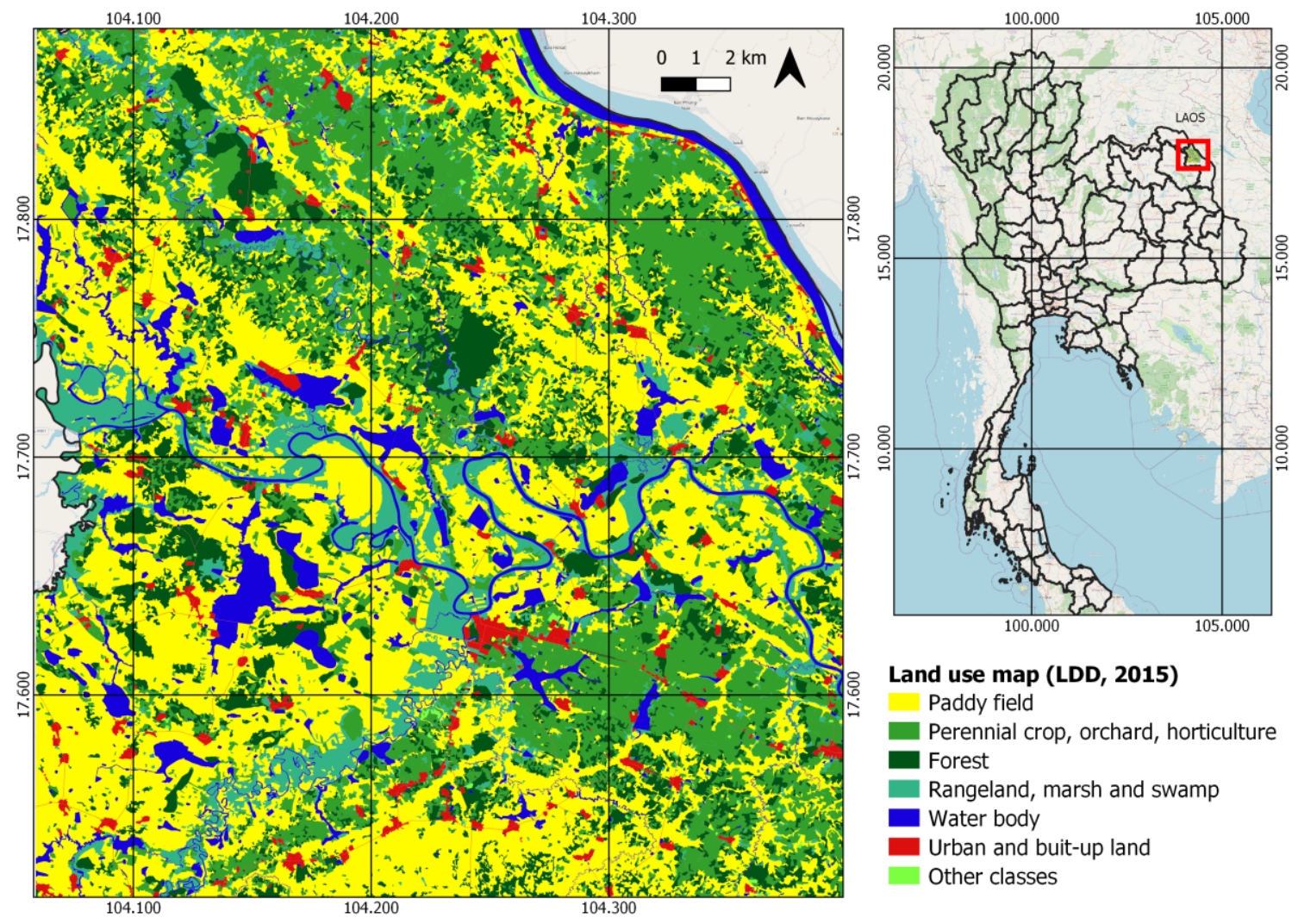

Land use map (LDD, 2015) Paddy field

Perennial crop, orchard, horticulture

- Forest

Rangeland, marsh and swamp

Water body

Urban and buit-up land

Other classes

Figure 1: Study area information from Land Development Department of Thailand (LDD, 2015) 
Table 1: Data used in this study

\begin{tabular}{|c|c|c|c|}
\hline \multicolumn{4}{|c|}{ Analysis data } \\
\hline Sensors & Situation & Date & \multirow{2}{*}{ Type } \\
\hline \multirow{2}{*}{ ALOS-2 } & before flood & 06-Apr-18 & \multirow{2}{*}{ SAR } \\
\cline { 2 - 3 } & during flood & 10 -Aug-18 & \multirow{2}{*}{ SAR } \\
\hline \multirow{2}{*}{ Sentinel-1 } & before flood & 01-Apr-18 & \multirow{2}{*}{ Optical } \\
\cline { 2 - 3 } & during flood & 05-Aug-18 & \multirow{2}{*}{ Reference information } \\
\hline \multirow{2}{*}{ Sentinel-2 } & before flood & 09-Apr-18 & 07-Aug-18 \\
\cline { 2 - 3 } & during flood & &
\end{tabular}
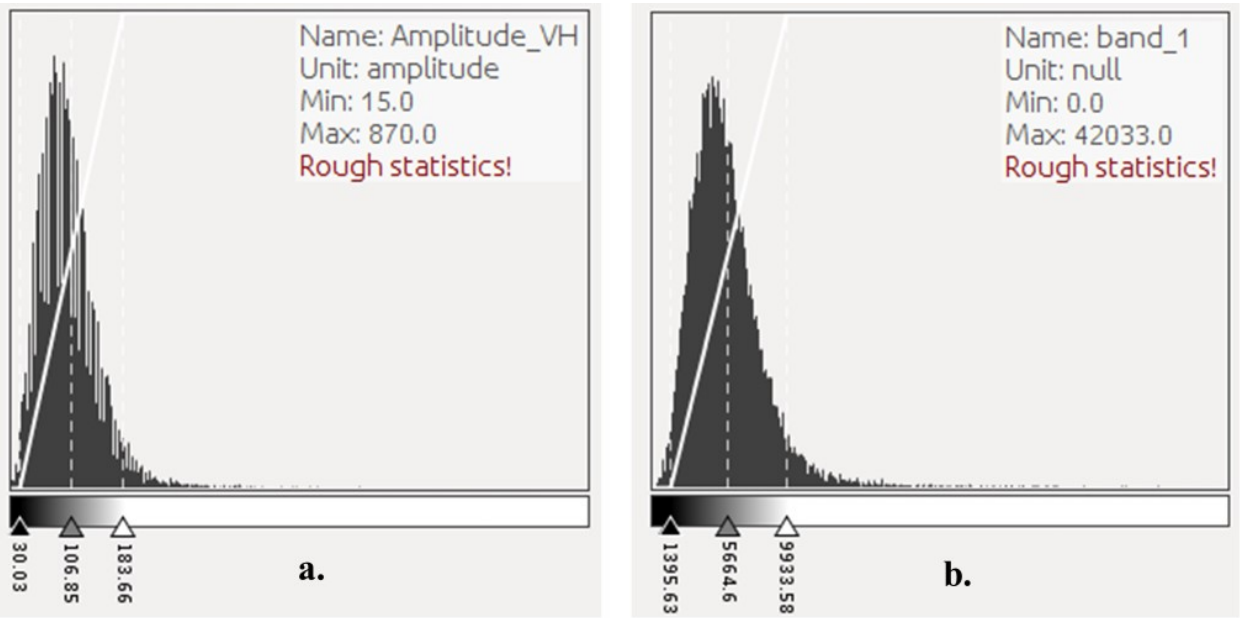

Figure 2: Digital number of Sentinel-1 and ALOS-2. Image a. corresponds to VH polarization of Sentinel-1, and image $b$. is an HH image of ALOS-2

This mode has a wide swath $(250 \mathrm{~km}$ swath $)$ and $5 \times 20 \mathrm{~m}$ spatial resolution. The study used ground range detected geo-referenced products (Level-1 or GRD) for analysis, and optical images of Sentinel-2 for accuracy assessment. Additionally, the researchers used free software including SNAP for SAR calibration, OpenCV for thresholding and QGIS for analysis and mapping.

\subsection{Data Pre-Processing}

SAR data (high-level product - raw pixel value) are presented in terms of digital number (DN) or intensity of backscattering energy from the earth's surface. DN can also be directly analyzed via the thresholding method, but the ranges of intensity vary regarding different sensors. In Figure 2, the ALOS-2 DN is put between $0-42000$ and the Sentinel-1 DN between 0-900, depending on each instance. ALOS-2 and Sentinel-1 were converted to backscattering properties in decibel units to be more useful and comparable between sensors. First, the ALOS-2 image was calibrated from digital number (DN) to backscattering (Sigma 0). Digital number in the PALSAR-2 JAXA standard product was converted to backscattering coefficient (sigma naught, sigma zero) (JAXA, 2019) using Equation 1.

$$
\sigma^{0}(d B)=10 \cdot \log _{10}\left\langle D N^{2}\right\rangle+C F_{1}
$$

Equation 1

$\sigma^{0}$ : Backscattering coefficient (Sigma naught or Sigma zero) [unit: $\mathrm{dB}$ ]

DN: Digital number

$\mathrm{CF}_{1}$ : Calibration factor [unit: $\left.\mathrm{dB}\right](\mathrm{CF} 1$ is -83$)$

For the calibration of Sentinel-1, the GRD product was used and the radiometric calibration $\sigma_{i}^{0}$ was applied via the information provided in the calibration lookup table (LUT) (ESA, 2019). The radiometric calibration was applied by Equation 2 .

$$
\text { value }(i)=\frac{\left|D N_{i}\right|^{2}}{A_{i}^{2}}
$$

Equation 2

Here, depending on the selected LUT, Value (i) is one of $\beta_{i}^{0}, \sigma_{i}^{0}$ or $\gamma_{i}$ or original $D N_{i} . \mathrm{A}_{\mathrm{i}}$ is one of betaNought $(i)$, sigmaNought(i), gamma(i) or $d n(i)$. 
Then, Equation 3 converted value (i) to decibel unit.

$$
\sigma^{0}(d B)=10 \cdot \log _{10}\left\langle\sigma_{i}^{0}\right\rangle
$$

Equation 3

\subsection{Water Thresholding and Detection}

This study aimed to assess two different sensors (Lband and C-band) for water and flood detection. Thresholding is the most rapid classification of a binary image. The Otsu method entails thresholding methods and was used in this study as it can be automatically investigated in the detection of water areas. Moreover, the Otsu algorithm performed powerfully in mapping inundated areas during flood events in Kerala, India, in 2018 (Tiwari et al., 2020).

The automatic image thresholding was performed by calculating a probability distribution over pixel intensities and finding the optimal threshold. The threshold value is determined by the minimum within-class variance or the maximum between-class variance (Otsu, 1979 and Huang et al., 2011). Good samples for Otsu thresholding should present a bimodal distribution (histogram with two peak distributions), and this method investigates the optimal threshold between two peaks. This study selected regions of interest (ROI) that included half area of water and non-water to calculate for local threshold value in 50 samples (Figure 3). Next, the mean threshold value was adopted for each image.

This study applied Lee-sigma to reduce SAR speckle noise. It compared two sensors, including in ALOS-2 and Sentinel-1, with non-filter and filter applications by considering these variables:

- Independent variable: Input of non-filtered and filtered ALOS-2 (HH, HV) and Sentinel-1 (VV, VH) images
- Control variable: Otsu method, same training areas (ROI) for thresholding calculation regarding each group in "before flood" and "during flood" (about 50 samples)

- Dependent variable: output of different threshold values and map results

At least two images could distinguish between permanent water bodies and floodwaters. This study utilized water thresholding extensions towards flood detection by images from events pre- ("before") and mid- ("during") flood.

\subsection{Accuracy Assessment}

Accuracy assessment was evaluated by optical images of pre- and mid-flood events. Reference water and non-water areas were detected by the supervised classification method.

Sentinel-2 has been used efficiently in landcover mapping (Chymyrov et al., 2018). Therefore, this study selected Sentinel-2 to evaluate the results of the SAR analysis. The related classifications were "water," "non-water," and "other," made via maximum-likelihood algorithms using a semiautomatic classification plugin (SCP) installed in QGIS (Congedo, 2019). Thresholding methods divided results into classes including "water" and "non-water" areas for SAR images. Thus, other classes (Sentinel-2) which do not fall within these two classes ("cloud" and "unclassified") were grouped into "no-data" (black color) and excluded from this accuracy assessment (Figure 4). The accuracy was performed as "overall accuracy." Water detection accuracy from pre- and mid-flood events was considered as "overall multi-temporal accuracy."

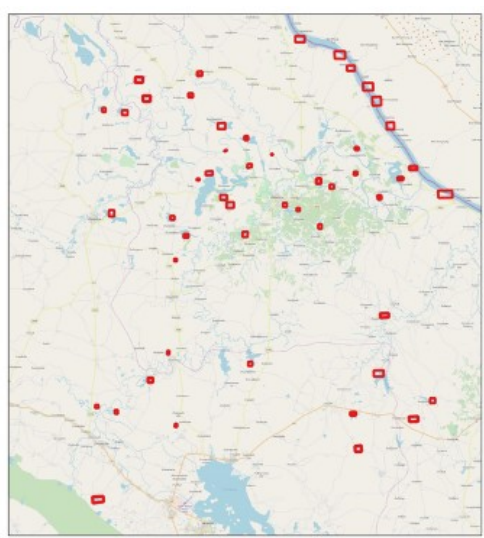

ROI for before-flood image

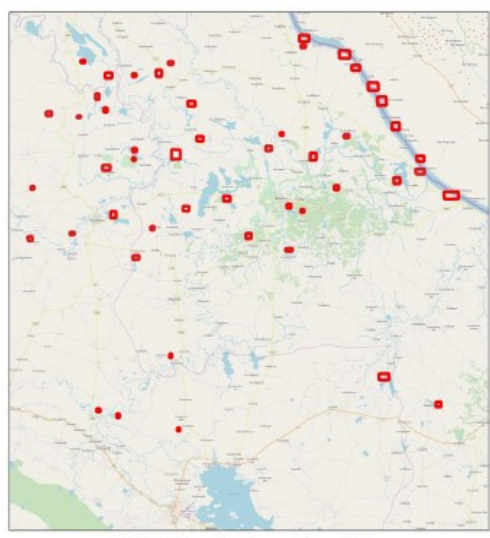

ROI for during-flood image
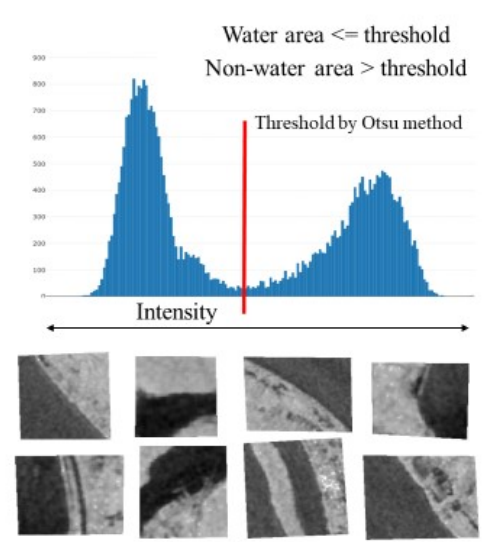

ROI example

Figure 3: Samples of "before flood" and "during flood" ROI for determining threshold value 


\section{Results}

ALOS-2 and Sentinel-1 could detect water and flood in the rainy season with less atmospheric effects compared to the Sentinel-2 optical sensor. These satellite images were observed in the same areas and in "near-time" acquisition to compare characteristics and differences. Two SAR images ("before" and "during" flood) were implemented for water and flood detection using the Otsu method. All results provided high accuracy: more than $85 \%$ compared to Sentinel-2. However, each sensor provided different characteristics for water and flood detection.

\subsection{Threshold Value in Decibel unit Using ALOS-2 and Sentinel-1 for Water Extraction}

After SAR pre-processing, the water threshold, in decibels, was identified by the Otsu method. Different sensors and polarizations had different thresholding values, as shown in "Table 2: Water threshold value for ALOS-2" and "Table 3: Water threshold value for Sentinel-1." Water areas were delineated by the Otsu thresholding of 50 samples.

Images of pre- and mid-flood events could come to present flood extension. The water area presented by SAR images had low backscattering, between 25 and $-15 \mathrm{~dB}$ for all polarizations. Co-polarized images of ALOS-2 $\mathrm{HH}$ and Sentinel-1 VV had a higher threshold value than cross-polarized images of ALOS-2 HV and Sentinel-1 VH.
3.2 Extension of Water Thresholding to Flood Detection by Images "Before" and "During" Flood Seasonal conditions affected the backscatter thresholding value in classifying "water" and "nonwater." Therefore, mean threshold values (from Tables 2 and 3) were applied to each temporal image, as shown in Figure 5 and Figure 6. The results demonstrated that ALOS-2 and Sentinel-1 can be used to extract open-water bodies with good agreement to water extraction, using Sentinel-2 images by supervised classification (Figure 4). The accuracy of filtered images slightly improved. The results of non-filtered images displayed a signal noise higher than the filtered images.

\subsection{Accuracy Assessment of ALOS-2 and Sentinel-1} for Water and Flood Detection

All results were compared to Sentinel-2, with water and non-water areas being detected for two periods: "before flooding" and "during flood" (Figure 4). Overall accuracy was calculated as shown in Table 4 and Table 5. The results of filtered images exhibited higher accuracy than those of non-filtered images. For ALOS-2, the HH image offered higher accuracy $(92.46 \%)$ than the HV for flood detection. The VV image of Sentinel-1 displayed the highest accuracy level, at $95.45 \%$, not only for its product (VH) but also at a level higher than the $\mathrm{HH}$ and $\mathrm{HV}$ images of ALOS-2 results, as demonstrated here.

Table 2: Water threshold value of ALOS-2

\begin{tabular}{|c|c|c|c|c|c|}
\hline \multirow{2}{*}{ Filter } & \multirow{2}{*}{ Bands } & \multicolumn{2}{|c|}{ Water before flooding } & \multicolumn{2}{c|}{ Water during flooding } \\
\cline { 3 - 6 } & & mean (dB) & $\begin{array}{c}\text { Standard } \\
\text { deviation }\end{array}$ & mean (dB) & $\begin{array}{c}\text { Standard } \\
\text { deviation }\end{array}$ \\
\hline \multirow{2}{*}{ Non-filtered } & HH & -16.97 & 1.91 & -16.48 & 1.87 \\
\cline { 2 - 6 } & HV & -24.86 & 1.83 & -24.11 & 1.97 \\
\hline \multirow{2}{*}{ Filtered } & HH & -16.21 & 2.05 & -15.66 & 1.49 \\
\cline { 2 - 6 } & HV & -24.29 & 1.66 & -23.61 & 1.78 \\
\hline
\end{tabular}

Table 3: Water threshold value of Sentinel-1

\begin{tabular}{|c|c|c|c|c|c|}
\hline \multirow{2}{*}{ Filtered } & \multirow{2}{*}{ Bands } & \multicolumn{2}{|c|}{ Water before flooding } & \multicolumn{2}{c|}{ Water during flooding } \\
\cline { 3 - 6 } & & mean (dB) & $\begin{array}{c}\text { Standard } \\
\text { deviation }\end{array}$ & mean (dB) & $\begin{array}{c}\text { Standard } \\
\text { deviation }\end{array}$ \\
\hline \multirow{2}{*}{ Non-filtered } & $\mathrm{VH}$ & -20.40 & 0.93 & -21.05 & 0.78 \\
\cline { 2 - 6 } & $\mathrm{VV}$ & -15.98 & 0.89 & -15.24 & 1.17 \\
\hline \multirow{2}{*}{ Filtered } & $\mathrm{VH}$ & -20.02 & 0.80 & -20.64 & 0.68 \\
\cline { 2 - 6 } & $\mathrm{VV}$ & -15.73 & 1.00 & -14.67 & 0.91 \\
\hline
\end{tabular}




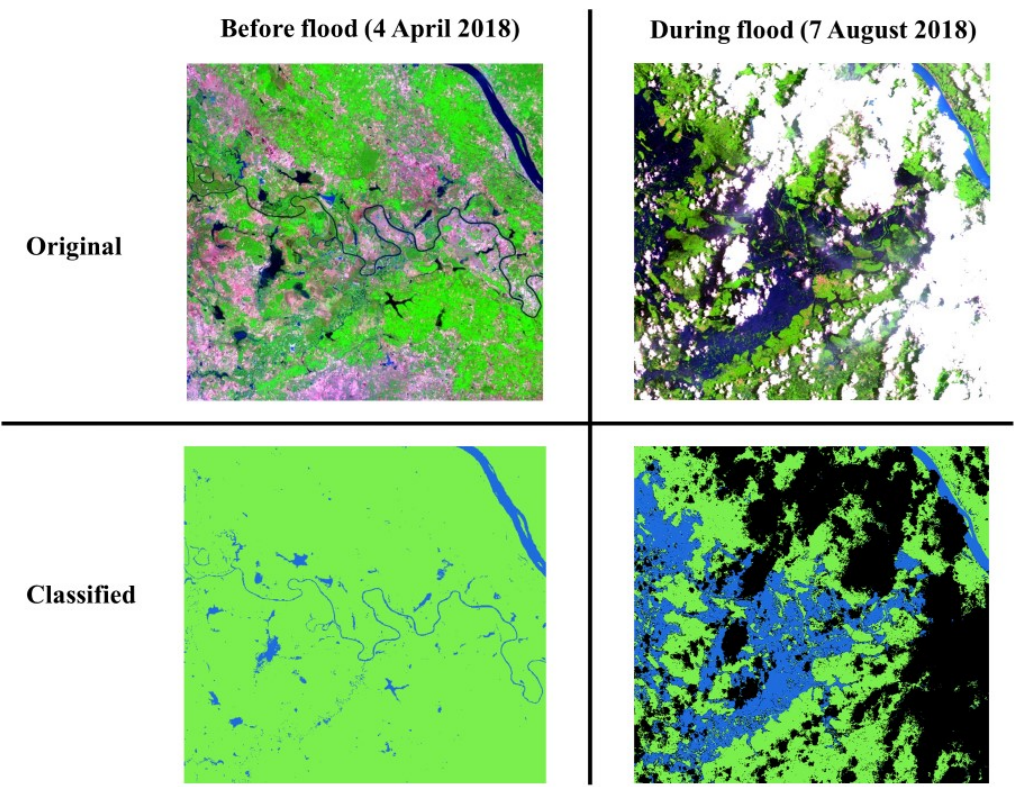

Non-water area Water area

No data (cloud/ unclassified classes)

Figure 4: Water and non-water classification using Sentinel-2 images
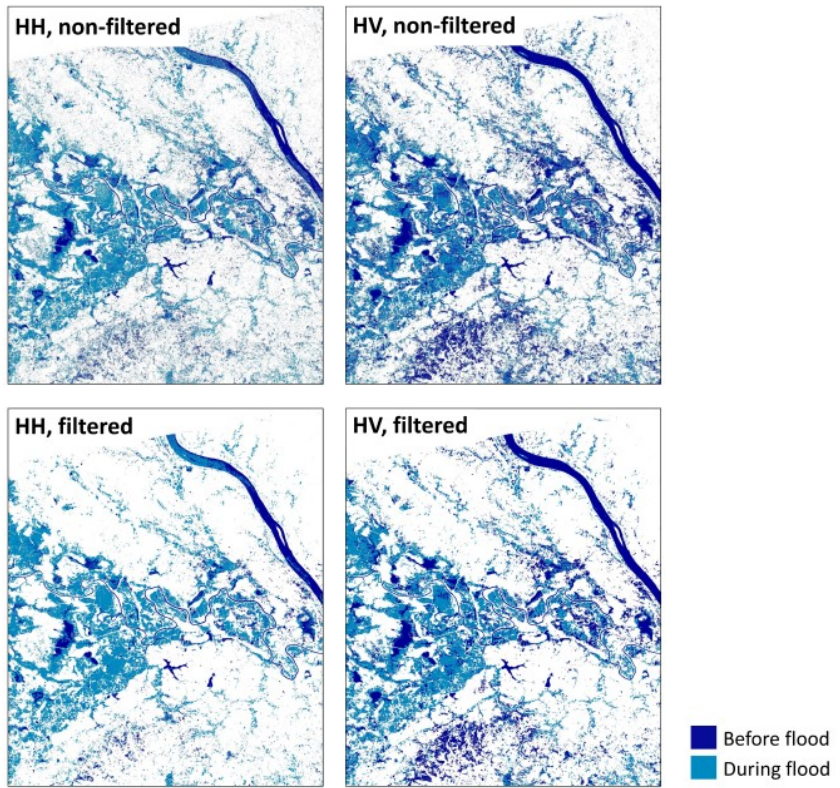

Figure 5: Flood detection using ALOS-2 images before and during flood

Table 4: Accuracy Assessment for water extraction between Sentinel-2 and ALOS-2

\begin{tabular}{|c|c|c|c|c|}
\hline \multirow{2}{*}{ Filter } & Bands & Before flood & During flood & \multirow{2}{*}{$\begin{array}{c}\text { Multi-temporal } \\
\text { accuracy (average) }\end{array}$} \\
\cline { 3 - 4 } & & Accuracy & Accuracy & $89.54 \%$ \\
\hline ALOS-2 & HH & $93.01 \%$ & $86.06 \%$ & $86.36 \%$ \\
\cline { 2 - 4 } Non-filtered & HV & $87.43 \%$ & $85.29 \%$ & $\mathbf{9 2 . 4 6 \%}$ \\
\hline ALOS-2 & HH & $96.38 \%$ & $88.55 \%$ & $89.26 \%$ \\
\cline { 2 - 4 } Filtered & HV & $91.53 \%$ & $87.00 \%$ & \\
\hline
\end{tabular}


Table 5: Accuracy Assessment for water extraction between Sentinel-2 and Sentinel-1

\begin{tabular}{|c|c|c|c|c|}
\hline Filter & Bands & Before flood & During flood & $\begin{array}{c}\text { Multi-temporal } \\
\text { accuracy (average) }\end{array}$ \\
\cline { 3 - 4 } & & Accuracy & Accuracy & $90.10 \%$ \\
\hline Sentinel-1 & VH & $88.91 \%$ & $91.29 \%$ & $94.05 \%$ \\
\cline { 2 - 4 } Non-filtered & VV & $95.95 \%$ & $92.14 \%$ & $92.88 \%$ \\
\hline Sentinel-1 & VH & $93.55 \%$ & $92.20 \%$ & $\mathbf{9 5 . 4 5 \%}$ \\
\cline { 2 - 4 } Filtered & VV & $98.16 \%$ & $92.73 \%$ & \\
\hline
\end{tabular}
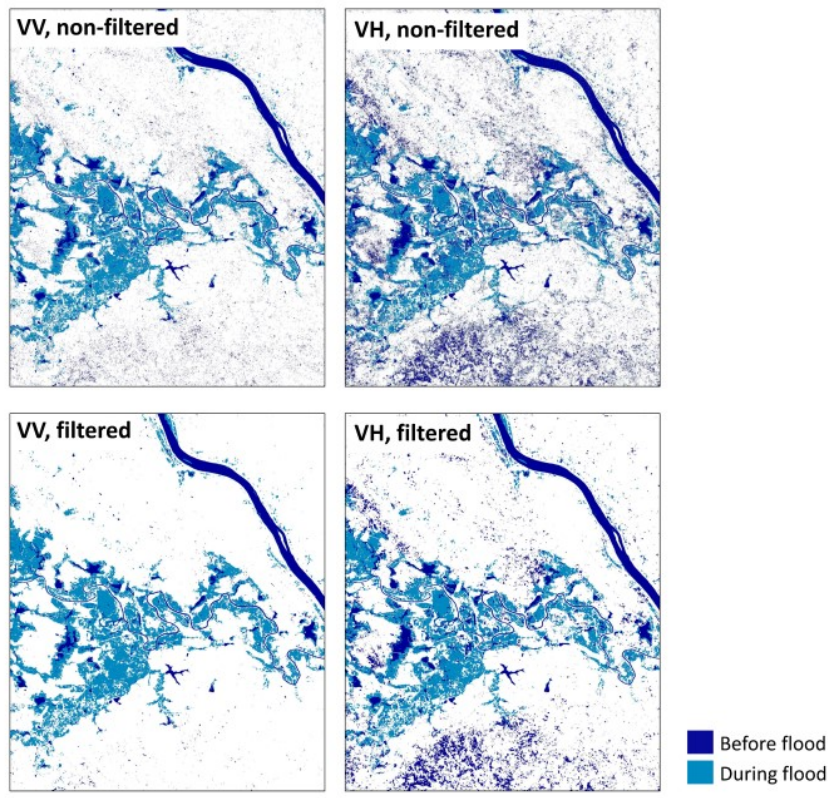

Figure 6: Flood detection using Sentinel-1 images from before and during flood

Table 6: Comparison between ALOS-2 HH and Sentinel-1 VV for water extraction (“during-flood” images)

\begin{tabular}{|c|c|c|c|}
\hline \multicolumn{2}{|c|}{ Similarity and difference } & \multicolumn{2}{c|}{ ALOS-2 HH } \\
\cline { 2 - 4 } & Non-water & $71.41 \%$ (similar) & $2.68 \%$ (different - B) \\
\hline \multirow{2}{*}{ Sentinel-1 VV } & Water & $6.12 \%$ (different - A) & $19.79 \%$ (similar) \\
\cline { 2 - 4 } &
\end{tabular}

Table 7: Land use types of different water detection between ALOS-2 and Sentinel-1

\begin{tabular}{|c|c|c|c|c|}
\hline Land use types (LDD, 2015) & $\begin{array}{c}\text { Different - A } \\
\text { (sq.km) }\end{array}$ & $\begin{array}{c}\text { Different - B } \\
(\mathbf{s q . k m )}\end{array}$ & $\begin{array}{c}\text { Total } \\
(\mathbf{s q . k m})\end{array}$ & Percentage \\
\hline Paddy field & 73.31 & 18.12 & 91.43 & $77.75 \%$ \\
\hline Perennial crop, orchard, horticulture & 1.42 & 2.07 & 3.49 & $2.97 \%$ \\
\hline Forest & 0.20 & 0.32 & 0.52 & $0.44 \%$ \\
\hline Rangeland, marsh and swamp & 1.91 & 9.59 & 11.50 & $9.78 \%$ \\
\hline Water body & 5.90 & 4.52 & 10.42 & $8.86 \%$ \\
\hline Urban and built-up land & 0.17 & 0.08 & 0.25 & $0.21 \%$ \\
\hline
\end{tabular}



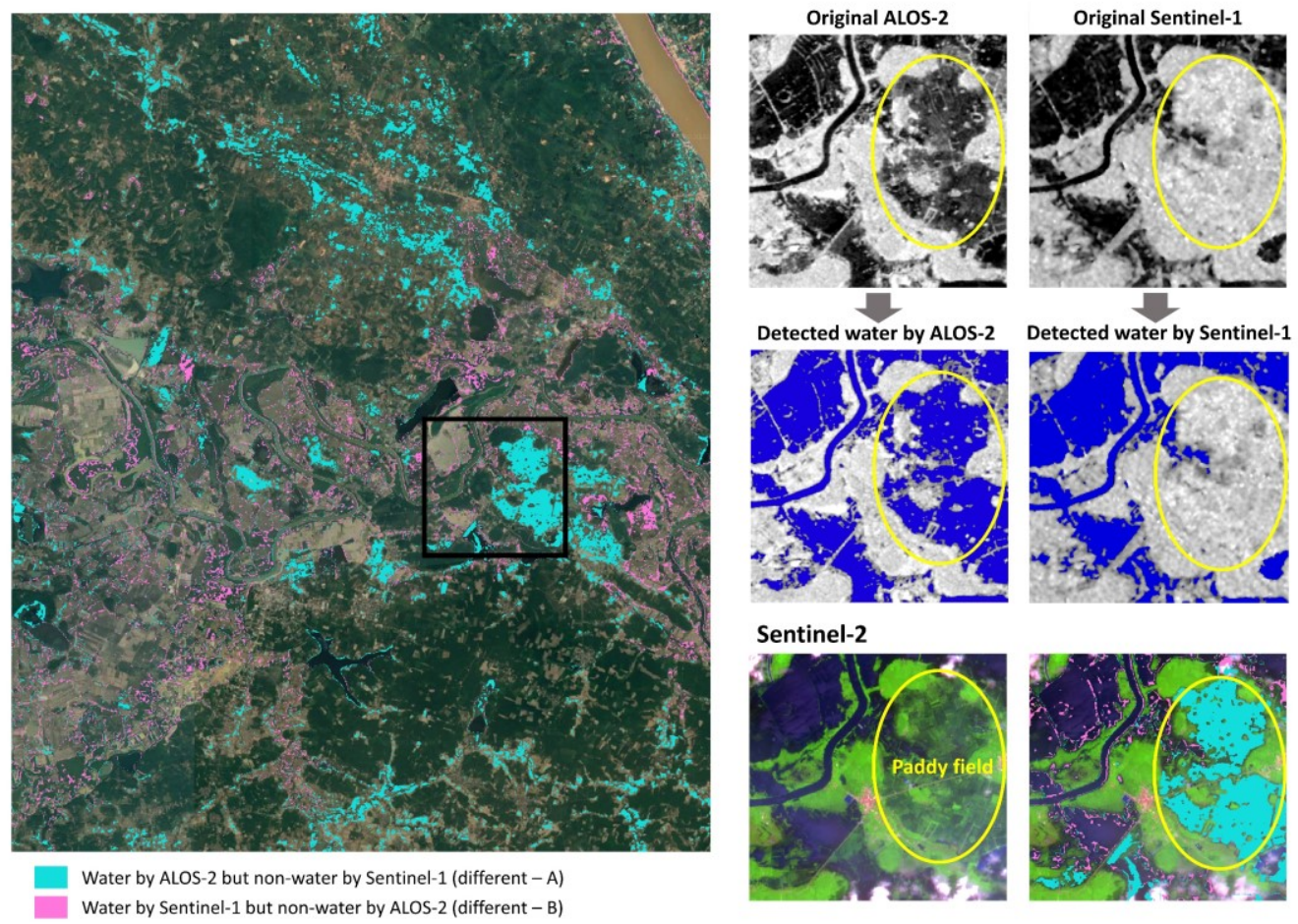

Figure 7: Different water detection areas between ALOS-2 and Sentinel-1

\subsection{Comparison between ALOS-2 L-band and}

Sentinel-1 C-band

Co-polarized images (ALOS-2 $\mathrm{HH}$ and Sentinel-1 VV) were compared for differences between these sensors as they provided the highest overall accuracy for each sensor. Based on "during-flood" images, similarity between ALOS-2 and Sentinel-1 was $91.2 \%(71.41 \%+19.79 \%)$ and difference was $8.8 \%(6.12 \%+2.68 \%)$ in this area, as presented in Table 6. The major difference for water area detection using the thresholding method was found in the paddy fields, at $77.75 \%$ of the detection between ALOS-2 and Sentinel-1, as shown in Table 7. The different L-band and C-band wavelengths were probably the main reason for this. For L-band, paddy fields appeared dark, the same as open water and flood areas. On the other hand, C-band's shortwavelength was more sensitive and reflected the energy atop the paddy fields, showing bright pixels (Figure 7).

\section{Discussion}

This study found that co-polarized images (ALOS-2 $\mathrm{HH}$ and Sentinel-1 VV) showed higher accuracy than cross-polarized images (ALOS-2 HV and Sentinel-1 VH) using Otsu thresholding. Sentinel-1 VV provided the highest overall accuracy.
The results corroborated previous research where co-polarization (Sentinel-1 VV) showed higher accuracy than cross-polarization (Sentinel-1 VH) for flood mapping using the thresholding method (Clement et al., 2018 and Cao et al., 2019). However, different detection methods may reveal different levels of accuracy for water body extraction.

Although the Sentinel-2 images compared with SAR images were not taken on the same dates, the observation dates were within three days of each other (Figure 8). Co-polarized images displayed higher accuracy compared to water extraction by Sentinel-2. Hence, results recommend the application of co-polarized images for the detection of open water and floodwater in emergency response.

Sentinel-1 VV revealed higher accuracy compared to ALOS-2 HH both pre- and mid-flood. This is likely due to the wavelength of Sentinel-1 $(5.6 \mathrm{~cm})$ being closer to Sentinel-2 $(443-2190 \mathrm{~nm})$ than ALOS-2 $(21 \mathrm{~cm})$. It is important to note, however, that this case pertained to long-term inundation; SAR utilization is more difficult and challenging during flash floods, since water quickly moves away from the affected area. 


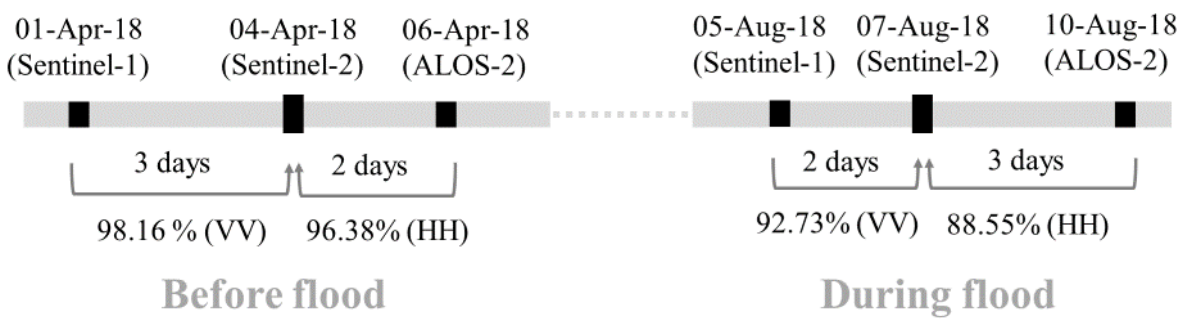

Figure 8: Comparison of SAR to Sentinel-2 and observation dates for water detection

The Otsu method was used to classify bimodal histograms into two classes ("open water" and "nonwater"). Nevertheless, flood behavior is not limited to reducing SAR backscattering: Some types of vegetative floods could, conversely, induce high backscattering (double-bound effects) depending on the wavelengths and surface conditions related to wetlands (Wohlfart et al., 2018). For that reason, vegetative floods could not be represented by a simple (two-class) thresholding method. Yet overall, the study found that the thresholding method could be used for rapid processing in emergency response efforts.

In the accuracy assessment, only "open water" and "non-water" (including paddy fields) were evaluated, using the Otsu method. Other vegetative flooding instances (of perennial crops, etc.) were excluded from this analysis. Hence, future studies should consider other advanced techniques for classifying water areas ("open water", "open floodwater" and water in vegetated areas).

Moreover, this study investigated only two satellite sensors. Other sensors should be analyzed and compared to gain knowledge on characteristics, physical properties and offset among different sensors. This could be useful for preliminary analysis in an urgent situation. Having additional multi-temporal images at hand would be more beneficial for monitoring flood dynamics and supporting disaster management. Considering multisensors could also increase the opportunities and speed in emergency response and disaster mapping.

\section{Conclusion}

This study attempted to gauge the ability to utilize ALOS-2 and Sentinel-1 images for water and flood detection. The thresholding method was used to determine the threshold values of backscattering coefficients for detecting water and flood areas in Nakhon Phanom province. The study was designed to compare filter effects ("filtered" and "nonfiltered") and the capacity of all polarization images for ALOS-2 and Sentinel-1. When compared with water and non-water area detection from Sentinel-2, Sentinel-1 VV has a higher overall accuracy level
$(95.45 \%)$ in this study area. Moreover, co-polarized images (ALOS-2 HH and Sentinel-1 VV) with filter application ("filtered") displayed higher accuracy than cross-polarized images (ALOS-2 HV and Sentinel-1 VH) for the same sensor. The comparison between ALOS-2 HH and Sentinel-1 VV indicated an $8.8 \%$ difference and mainly appeared on paddy fields (vegetative floods).

Based on these results, the study recommends the use of co-polarized images for water and flood detection via the thresholding method. These findings can be useful for disaster mapping and management. For future research, further imaging, methods and data fusion should be considered to improve the accuracy and speed in getting information about disasters.

\section{References}

Acharya, T. D., Lee, D. H., Yang, I. T. and Lee, J. K., 2016, Identification of Water Bodies in a Landsat 8 OLI Image Using a J48 Decision Tree. Sensors (Switzerland), Vol. 16(7), 1-16. https://doi.org/10.3390/s16071075.

ADRC., 2019, Details of Disaster Information. Retrieved January 21, 2019, from https://www.adrc.asia/view_disaster_en.php?Nat ionCode $=764 \&$ lang $=$ en $\&$ KEY $=2284$.

Cao, H., Zhang, H., Wang, C. and Zhang, B., 2019, Operational Flood Detection Using Sentinel-1 SAR Data Over Large Areas. Water (Switzerland), Vol. 11(4). https://doi.org/10.3390/w11040786.

Chymyrov, A., Betz, F., Baibagyshov, E., Kurban, A., Cyffka, B. and Halik, U., 2018, Landcover Mapping of Floodplains along the Naryn River, Kyrgyzstan Based on Sentinel-2. International Journal of Geoinformatics, Vol. 14(1), 21-28.

Clement, M. A., Kilsby, C. G. and Moore, P., 2018, Multi-Temporal Synthetic Aperture Radar Flood Mapping Using Change Detection. Journal of Flood Risk Management, Vol. 11(2), 152-168. https://doi.org/10.1111/jfr3.12303.

Congedo, L., 2019, Semi-Automatic Classification Plugin Documentation Release 6.2.0.1. Release. https://doi.org/10.13140/RG.2.2.29474.02242/1 
Duy, N. B., 2015, Automatic Detection of Surface water bodies from Sentinel-1 SAR Images Using Valley-Emphasis Method. Vietnam Journal of Earth Sciences, Vol. 37(4), 328-343. https://doi.org/10.15625/0866-7187/37/4/8298.

Eberhardt, I. D. R., Schultz, B., Rizzi, R., Sanches, I. D. A., Formaggio, A. R., Atzberger, C. and Luiz, A. J. B., 2016, Cloud Cover Assessment for Operational Crop Monitoring Systems in Tropical Areas. Remote Sensing, Vol. 8(3), 114. https://doi.org/10.3390/rs8030219.

ESA. 2019, Level-1 Radiometric Calibration Application of Radiometric Calibration LUT Application of De-Noise LUT. Retrieved January 21, 2019, from https://sentinel.esa.int/web/sentinel/radiometric-calibration-of-level-1products.

Huang, D. Y., Lin, T. W. and Hu, W. C., 2011, Automatic Multilevel Thresholding Based on Two-Stage Otsu's Method with Cluster Determination by Valley Estimation. International Journal of Innovative Computing, Information and Control, Vol. 7(10), 5631-5644.

JAXA, 2019, Calibration Result of ALOS-2 / PALSAR-2 JAXA Standard Products. Retrieved January 21, 2019, from https://www.eorc.jaxa.jp/ALOS-2/en/calval/cal-val_index.htm.

Kaku, K. and Held, A., 2013, Sentinel Asia: A Space-Based Disaster Management Support System in the Asia-Pacific Region. International Journal of Disaster Risk Reduction, Vol. 6, 1-17. https://doi.org/10.1016/j.ijdrr.2013.08.004.

Kanta Kafle, S., 2017, Disaster Risk Management Systems in South Asia: Natural Hazards, Vulnerability, Disaster Risk and Legislative and Institutional Frameworks. Journal of Geography \& Natural Disasters, Vol. 7(3). https://doi.org/10.4172/2167-0587.1000207.

Land Development Department. Land Use Map 2015; Ministry of Agriculture and Cooperatives: Bangkok, Thailand, 2015

Miyazaki, H., Nagai, M. and Shibasaki, R., 2015, Reviews of Geospatial Information Technology and Collaborative Data Delivery for Disaster Risk Management. ISPRS International Journal of Geo-Information, Vol. 4(4), 1936-1964. https://doi.org/10.3390/ijgi4041936.

Nakmuenwai, P., Yamazaki, F. and Liu, W., 2017, Automated Extraction of Inundated Areas from Multi-temporal Dual-Polarization Radarsat-2 Images of the 2011 Central Thailand flood. Remote Sensing, Vol. 9(1), 1-19. https://doi.org/10.3390/rs9010078.

Otsu, N., 1979, A Tlreshold Selection Method from Gray-Level Histograms. IEEE Transactions on Systems, Man, and Cybernetics, Vol. 9(1), 62-
66. https://doi.org/10.1109/TSMC.1979.4310076.

Plank, S., Jüssi, M., Martinis, S. and Twele, A., 2017, Mapping of Flooded Vegetation by Means of Polarimetric Sentinel-1 and ALOS2/PALSAR-2 Imagery. International Journal of Remote Sensing, Vol. 38(13), 3831-3850. https://doi.org/10.1080/01431161.2017.1306143

Stevens, D., 2008, Increasing the Use of Geospatial Technologies for Emergency Response and Disaster Rehabilitation in Developing Countries. In: Nayak S., Zlatanova S. (eds) Remote Sensing and GIS Technologies for Monitoring and Prediction of Disasters. Environmental Science and Engineering (Environmental Science). Springer, Berlin, Heidelberg. https://doi.org/10.1007/978-3-540-79259-8_4

Tamkuan, N. and Nagai, M., 2017, Fusion of MultiTemporal Interferometric Coherence and Optical Image Data for the 2016 Kumamoto Earthquake Damage Assessment. ISPRS International Journal of Geo-Information, Vol. 6(7), 188. https://doi.org/10.3390/ijgi6070188.

Tamkuan, N. and Nagai, M., 2019a, Damage Assessment Using ALOS-2/PALSAR-2 for the 2018 Earthquake in Palu, Sulawesi, Indonesia. 40th Asian Conference on Remote Sensing: Progress of Remote Sensing Technology for Smart Future, ACRS 2019; Daejeon Convention Center (DCC)Daejeon; South Korea.

Tamkuan, N. and Nagai, M., 2019b, Sentinel-1A Analysis for Damage Assessment: A Case Study of Kumamoto Earthquake In 2016. MATTER: International Journal of Science and Technology, Vol. 5(1), 23-35. https://doi.org/10.20319/mijst.2019.51.2335.

Tiwari, V., Kumar, V., Matin, M. A., Thapa, A., Ellenburg, W. L., Gupta, N. and Thapa, S., 2020, Flood inundation Mapping- Kerala 2018; Harnessing the Power of SAR, Automatic Threshold Detection Method and Google Earth Engine. PloS One, Vol. 15(8), e0237324. https://doi.org/10.1371/journal.pone.0237324

Wohlfart, C., Winkler, K., Wendleder, A. and Roth, A., 2018, TerraSAR-X and Wetlands: A review. Remote Sensing, Vol. 10(6), 1-24. https://doi.org/10.3390/rs10060916.

Xie, H., Luo, X., Xu, X., Pan, H. and Tong, X., 2016, Automated Subpixel Surface Water Mapping from Heterogeneous Urban Environments using Landsat 8 OLI Imagery. Remote Sensing, Vol. 8(7), 1-16. https://doi.org/10.3390/rs8070584 